\title{
Efficacy and safety of apatinib combined with chemotherapy for the treatment of advanced gastric cancer in the Chinese population: a systematic review and meta-analysis
}

This article was published in the following Dove Press journal:

Drug Design, Development and Therapy

\section{Honggang Cheng' \\ Aixia Sun ${ }^{2}$ \\ Qingbo Guo ${ }^{3}$ \\ Yucai Zhang ${ }^{4}$}

'Department of Gastroenterological Surgery, Liaocheng People's Hospital, Liaocheng Clinical School of Taishan Medical University, Liaocheng 252000 , Shandong Province, People's Republic of China; ${ }^{2}$ Department of Clinical Laboratory, Liaocheng People's Hospital, Liaocheng Clinical School of Taishan Medical University, Liaocheng 252000, Shandong Province, People's Republic of China; ${ }^{3}$ Department of Clinical Laboratory, Yidu Central Hospital of Weifang, Qingzhou 262500, Shandong Province, People's Republic of China; ${ }^{4}$ Department of Health, Liaocheng People's Hospital of Taishan Medical University, Liaocheng 252000, Shandong Province, People's Republic of China
Correspondence: Yucai Zhang Department of Health, Liaocheng People's Hospital of Taishan Medical University, Dongchang West Road 67, Liaocheng 252000, Shandong Province, People's Republic of China

Tel +86 I88 $6527 \quad 1910$

Email mmzhangyc@I63.com
Objective: To systematically evaluate the efficacy and safety of the combination of apatinib targeted therapy and chemotherapy (CT) in the treatment of patients with advanced gastric cancer (GC).

Materials and methods: Clinical trials were extracted from PubMed, the Cochrane Library, Web of Science, EMBASE, CNKI, and the Wanfang database. Outcome measures, including therapeutic efficacy, quality of life (QOL), and adverse events, were extracted and evaluated. Results: Nineteen trials, including 1,256 advanced GC patients, were included. The results indicated that, compared with CT alone, the combination of apatinib targeted therapy with CT significantly improved the patients' complete response rate $(\mathrm{OR}=1.85,95 \% \mathrm{CI}=1.04-3.28$, $P=0.04)$, partial response rate $(\mathrm{OR}=2.19,95 \% \mathrm{CI}=1.71-2.80, P<0.00001)$, overall response $(\mathrm{OR}=2.57,95 \% \mathrm{CI}=1.99-3.32, P<0.00001)$, and disease control rate $(\mathrm{OR}=3.46,95 \%$ $\mathrm{CI}=2.57-4.66, P<0.00001)$. Moreover, the combined therapy exhibited advantages over $\mathrm{CT}$ alone in the patients' QOL including the QOL improved rate $(\mathrm{OR}=1.77,95 \% \mathrm{CI}=0.94-3.33$, $P=0.08)$ and the Karnofsky performance score $(\mathrm{OR}=1.77,95 \% \mathrm{CI}=0.94-3.33, P=0.08)$. The group that received the combined therapy had higher rates of hypertension $(\mathrm{OR}=5.75,95 \%$ $\mathrm{CI}=2.22-14.92, P=0.0003)$, albuminuria $(\mathrm{OR}=15.42,95 \% \mathrm{CI}=5.39-44.10, P<0.00001)$, and hand-foot syndrome $(\mathrm{OR}=2.09,95 \% \mathrm{CI}=1.26-3.48, P=0.004)$, whereas analyses of other adverse events, such as leucopenia, thrombocytopenia, and neutropenia, did not reveal significant differences $(P>0.05)$.

Conclusion: The combination of apatinib targeted therapy and CT is more effective for GC treatment than CT alone. However, this combined treatment could lead to greater rates of hypertension, albuminuria, and hand-foot syndrome. Therefore, the benefits and risks should be considered before treatment.

Keywords: apatinib, target therapy, chemotherapy, gastric cancer, meta-analysis

\section{Introduction}

Gastric cancer (GC) is considered the third-leading cause of death among all cancer types and has caused 723,000 deaths across the whole world. ${ }^{1,2}$ Currently, the incidence of GC has been significantly increasing, with $\sim 952,000$ new cases each year. ${ }^{2}$ The People's Republic of China is a high-risk area for GC, and the new cases of GC in this region account for $\sim 42.5 \%$ of such cases in the world. ${ }^{3}$ Early GC can easily be misdiagnosed because of the small number of symptoms. Most patients with GC have developed to an advanced stage or exhibited metastasis, and the 5-year survival 
rate is $<20 \%$ at this stage. ${ }^{1,4}$ Chemotherapy (CT) is one of the standard treatment regimens for advanced GC. Although CT improves patient survival, most patients eventually relapse and develop resistance to treatment, which is not able to completely eradicate small lesions and metastatic cells. ${ }^{1,5-7}$ Thus, more effective and safer treatments are urgently required.

In recent years, the use of molecular targeted therapy has been increasing rapidly, and this approach is considered as a powerful therapeutic method for cancer treatment. The agents used in targeted therapy can precisely identify and attack certain type of cancer cells based on mutations of genes and proteins. ${ }^{2}$ Moreover, little damage is done to normal cells; thus, molecular targeted therapy is also called a "biological missile." The clinical application of molecular targeted therapy for malignancies has been reported, and several studies have found that the combination of molecular targeted therapy and CT has better therapeutic effects than treatment with CT alone. ${ }^{8-13}$

Angiogenesis is essential for tumor growth and metastasis, and VEGF and its receptors (VEGFRs) play a crucial role in angiogenesis. ${ }^{14-16}$ VEGFR2 is one of 3 VEGFRs and plays a pivotal role in VEGF-mediated cancer angiogenesis. ${ }^{17}$ Apatinib is a novel antiangiogenic agent that specifically targets VEGFR2. This small molecule tyrosine kinase inhibitor was approved for the second-line treatment of advanced GC in the People's Republic of China in 2014. ${ }^{17,18}$ In several clinical trials, apatinib targeted therapy combined with CT exhibited more prominent therapeutic effects for advanced GC than CT alone. ${ }^{19-37}$ However, systematic analyses assessing the therapeutic efficacy of apatinib targeted therapy combined with $\mathrm{CT}$ in advanced GC remain scarce. In this study, we conducted a meta-analysis to investigate the treatment effect and safety of apatinib targeted therapy combined with $\mathrm{CT}$ in comparison with $\mathrm{CT}$ alone for advanced GC to provide a scientific reference for the design of future clinical trials.

\section{Materials and methods}

\section{Search strategy and selection criteria}

The literature was searched across PubMed, the Cochrane Library, Web of Science, EMBASE, CNKI, and the Wanfang database with key terms "apatinib" combined with "gastric cancer." No language limits were applied. The initial search was performed in January 2018 and updated in March 2018.

Selection criteria: Studies concerning advanced GC patients were involved in our analysis. Patients in the experimental groups received apatinib targeted therapy combined with $\mathrm{CT}$, and patients in the control group were treated with $\mathrm{CT}$ alone.

\section{Data extraction and quality assessment}

The data were independently extracted by 2 investigators (Honggang Cheng and Aixia Sun). Disagreements were resolved by discussion with a third researcher (Qingbo Guo). All involved studies were summarized as follows: the first author's name, year of publication, study location, tumor stages, number of cases, patient ages, study parameter types, therapeutic regimens, enrollment period, and dosages of apatinib utilized. The included trials' qualities were evaluated based on the Cochrane Handbook. ${ }^{38}$

\section{Outcome definition}

The clinical responses included treatment efficacy, quality of life (QOL), and adverse events (AEs). Treatment efficacy was assessed in terms of the complete response rate (CR), partial response rate $(\mathrm{PR})$, stable disease rate $(\mathrm{SD})$, progressive disease rate $(\mathrm{PD})$, overall response rate $(\mathrm{ORR}, \mathrm{ORR}=\mathrm{CR}+\mathrm{PR})$, and disease control rate $(\mathrm{DCR}, \mathrm{DCR}=\mathrm{CR}+\mathrm{PR}+\mathrm{SD})$. The patients' QOLs were evaluated using the QOL improved rate (QIR) and the Karnofsky performance score (KPS). AEs, including leukopenia, thrombocytopenia, diarrhea, nausea and vomiting, hypertension, neutropenia, albuminuria, oral mucositis, hand-foot syndrome, weakness, hemoglobin reduction, and myelosuppression, were also assessed.

\section{Statistical analysis}

The analyses were performed using Review Manager 5.3 (Cochrane Collaboration, London, UK). $P<0.05$ was taken to indicate that the differences reached statistical significance. Heterogeneity among studies was assessed with the Cochran's $Q$ test to determine the most suitable analysis model, and funnel plots were used to assess the publication biases of the involved studies. ${ }^{39} I^{2}<50 \%$ or $P>0.1$ indicated that a study was homogenous. The OR was the principal measurement of the therapeutic effects, and the ORs are presented with the $95 \%$ CIs. Sensitivity analyses were conducted to evaluate the influences of the therapeutic regimens, apatinib dosages, sample sizes, and types of involved studies.

\section{Results}

\section{Search results}

A total of 476 articles were identified in the initial search. After review of the titles and abstracts, 384 articles were excluded because they did not include clinical trials $(n=207)$, 
were unrelated studies $(n=56)$, or were duplications or repetitions $(n=121)$. This process left 92 studies as potentially relevant. After detailed assessment of the full texts, case reports and reviews $(n=16)$, and articles without a control group $(n=17)$ or without apatinib and CT combined therapy $(n=23)$ or with insufficient data $(n=4)$ were excluded. Finally, 19 trials $^{19-37}$ involving 1,256 advanced GC patients were included in this meta-analysis (Figure 1).

\section{Patient characteristics}

After selection, all of the included trials were conducted in the People's Republic of China. In total, 625 advanced GC patients were treated with apatinib in combination with CT, and 631 patients were treated with CT alone. Detailed information about the involved trials and patients is presented in Tables 1 and 2.

\section{Quality assessment}

The assessment of bias risk is presented in Figure 2. Fourteen studies were determined to be low risk, and the remaining 5 studies were not true randomized controlled trials. None of the included trials provided clear descriptions of the performance and detection risks. The attrition risks of the involved trials were low; 3 trials were considered to have unclear risk owing to selective reporting.

\section{Therapeutic efficacy assessments}

As presented in Figures 3 and 4, Figure S1, and Table 3, the pooled results revealed that the patients who underwent combined therapy exhibited significantly improved CR, PR, ORR, and DCR (CR: OR=1.85, 95\% CI=1.04-3.28, $P=0.04$; PR: OR=2.19, 95\% CI=1.71-2.80, $P<0.00001$; ORR: $\mathrm{OR}=2.57,95 \% \mathrm{CI}=1.99-3.32, P<0.00001 ; \mathrm{DCR}: \mathrm{OR}=3.46$, $95 \% \mathrm{CI}=2.57-4.66, P<0.00001)$ and significantly decreased $\mathrm{PD}(\mathrm{OR}=0.33,95 \% \mathrm{CI}=0.25-0.44, P<0.00001)$, whereas the $\mathrm{SD}$ was not significantly different from that of the patients who received $\mathrm{CT}$ alone $(\mathrm{OR}=1.09,95 \% \mathrm{CI}=0.86-1.39$, $P=0.48$ ). Fixed effect models were used to analyze the OR rate because of low heterogeneity.

\section{QOL assessment}

QOL was evaluated in this analysis. The results revealed that the QOL of patients in the combined group was significantly better than that of the control group as indicated by the QIR and KPS, although the former did not reach significance (Figure 5, QIR: $\mathrm{OR}=1.77,95 \% \mathrm{CI}=0.94-3.33, P=0.08$; KPS: $\mathrm{OR}=14.99,95 \% \mathrm{CI}=12.51-17.47, P<0.00001)$.

\section{AEs assessment}

The safety of apatinib targeted therapy was evaluated in this meta-analysis. As presented in Figure S2 and

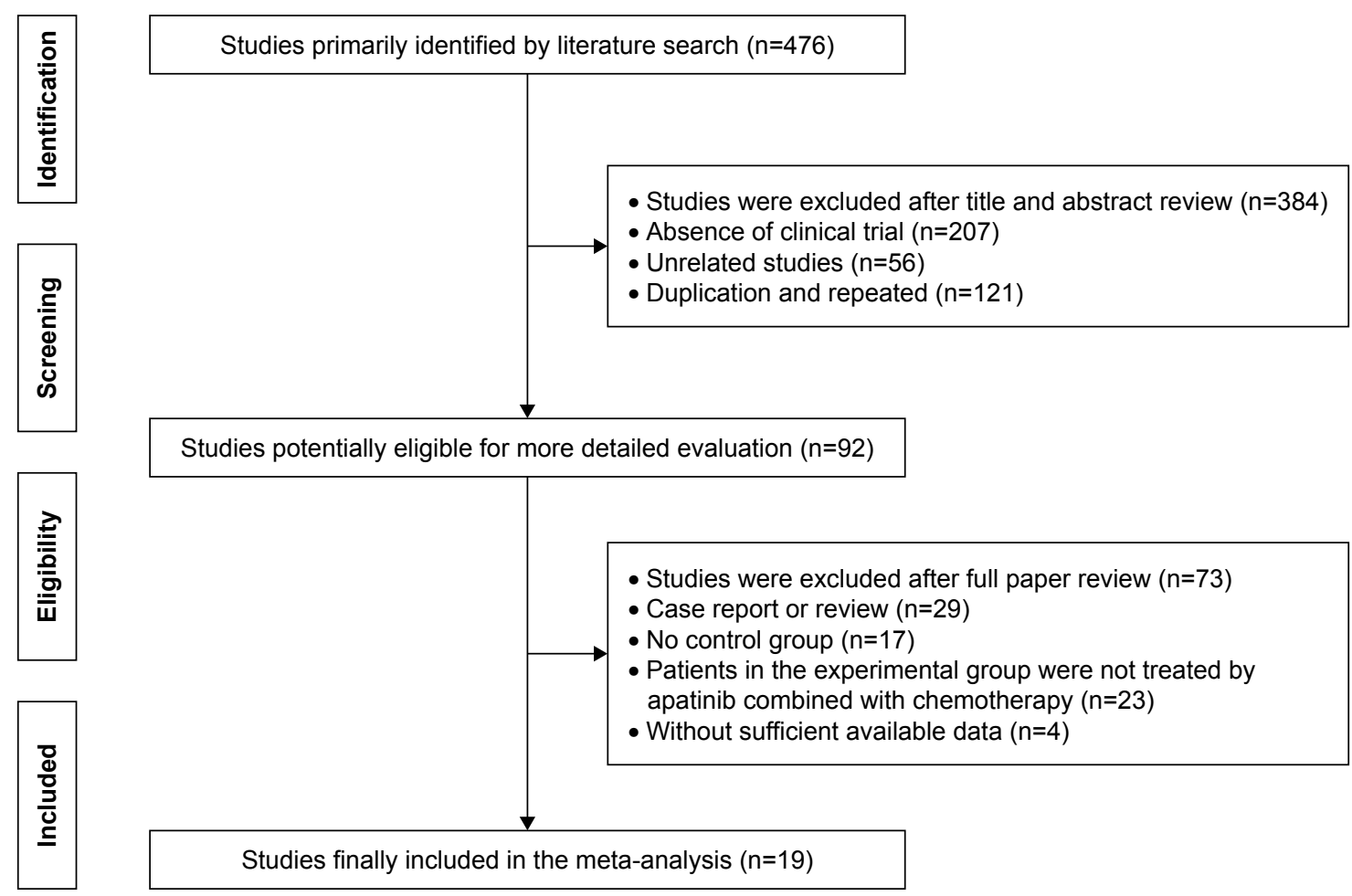

Figure I Flow diagram of the selection process. 
Table I Clinical information from the eligible trials in the meta-analysis

\begin{tabular}{|c|c|c|c|c|c|c|}
\hline \multirow[t]{2}{*}{ Included studies } & \multirow[t]{2}{*}{ Nation } & \multirow[t]{2}{*}{ Tumor stage } & \multirow{2}{*}{$\begin{array}{l}\text { Patients } \\
\text { Con/Exp }\end{array}$} & \multicolumn{2}{|l|}{ Age (year) } & \multirow[t]{2}{*}{ Parameter types } \\
\hline & & & & Con & Exp & \\
\hline Dong et al, ${ }^{19} 2018$ & People's Republic of China & ND & $4 I / 4 I$ & ND & ND & ORR, DCR, KPS, AE \\
\hline $\mathrm{Du},{ }^{20} 2017$ & People's Republic of China & 0-2 (ECOG) & $40 / 40$ & $54.6 \pm 10.4$ (mean) & $51.3 \pm 10.0$ (mean) & ORR, DCR, AE \\
\hline Duan et al, ${ }^{21} 2017$ & People's Republic of China & III-IV & $28 / 32$ & $64.7 \pm 9.9$ (mean) & $63.2 \pm 9.5$ (mean) & ORR, DCR, AE \\
\hline Fan et al, ${ }^{22} 2017$ & People's Republic of China & III-IV & $15 / 15$ & $\geq 70$ (I) & $\geq 70$ (2) & ORR, DCR, QIR, AE \\
\hline Gao et $\mathrm{al}^{23} 2017$ & People's Republic of China & 60-80 (KPS) & $15 / 16$ & ND & ND & ORR, DCR, AE \\
\hline Hu et al, ${ }^{24} 2016$ & People's Republic of China & ND & $23 / 23$ & $58.1 \pm 1.2$ (mean) & $57.8 \pm 1.1$ (mean) & ORR, DCR, AE \\
\hline Jing, ${ }^{25} 2016$ & People's Republic of China & 0-2 (ECOG) & $21 / 21$ & $75.1 \pm 3.7$ (mean) & $74.5 \pm 3.7$ (mean) & ORR, DCR, AE \\
\hline Li et al, ${ }^{26} 2018$ & People's Republic of China & IV & $30 / 34$ & $55.5 \pm 9.5$ (mean) & $56.0 \pm 10.7$ (mean) & ORR, DCR \\
\hline $\mathrm{Li}$ and $\mathrm{Li}^{27} 2017$ & People's Republic of China & $K P S \geq 60$ & $35 / 35$ & $54.3 \pm 1.8$ (mean) & $55.6 \pm 1.5$ (mean) & ORR, DCR, KPS \\
\hline $\mathrm{Li}^{28} 20 \mathrm{I} 7$ & People's Republic of China & 0-2 (ECOG) & $20 / 20$ & $48.0 \pm$ II.5 (mean) & $46.5 \pm 12.0$ (mean) & ORR, DCR, AE \\
\hline Qian and $\mathrm{Ge},{ }^{29} 2017$ & People's Republic of China & IV & $42 / 34$ & 61 (median) & 62 (median) & ORR, DCR, AE \\
\hline Sheng et al, ${ }^{30} 2017$ & People's Republic of China & III-IV & $59 / 59$ & $52.2 \pm 2.7$ (mean) & $51.4 \pm 2.6$ (mean) & ORR, DCR, AE \\
\hline Wang et al, ${ }^{31} 2016$ & People's Republic of China & ND & $29 / 29$ & $58.6 \pm 12.0$ (mean) & $57.2 \pm 8.3$ (mean) & ORR \\
\hline Wen et al, 2017 & People's Republic of China & III-IV & $45 / 45$ & $62.9 \pm 4.3$ (mean) & $63.3 \pm 4.2$ (mean) & ORR, DCR, AE \\
\hline Wu et al, ${ }^{33} 2017$ & People's Republic of China & III-IV & $14 / 14$ & $49.3 \pm 9.7$ (mean) & $48.9 \pm 9.8$ (mean) & ORR, DCR, QIR, AE \\
\hline Yan et al,,$^{34} 2017$ & People's Republic of China & IV & $75 / 75$ & $56 \pm 6$ (mean) & $54 \pm 6$ (mean) & ORR, DCR, AE \\
\hline Zhan, ${ }^{35} 2017$ & People's Republic of China & III-IV & $40 / 40$ & $63.7 \pm 3.4$ (mean) & $64.5 \pm 4.1$ (mean) & ORR, DCR, QIR, AE \\
\hline Zhou et al, ${ }^{36} 2018$ & People's Republic of China & ND & $20 / 20$ & $61.1 \pm 9.5$ (mean) & $60.2 \pm 8.7$ (mean) & ORR, DCR, QIR, AE \\
\hline Zhu et al, ${ }^{37} 2016$ & People's Republic of China & 0-2 (ECOG) & $39 / 32$ & 60 (median) & 56 (median) & ORR, DCR, AE \\
\hline
\end{tabular}

Notes: Con, control group (CT alone group); Exp, experimental group (apatinib targeted therapy plus CT)

Abbreviations: AE, adverse events; CT, chemotherapy; ECOG, Eastern Cooperative Oncology Group score; DCR, disease control rate; KPS, Karnofsky performance score; ND, nondetermined; ORR, overall response rate; QIR, quality of life improved rate.

Table 4, the group that received apatinib targeted therapy plus CT had higher rates of hypertension, albuminuria, and hand-foot syndrome (hypertension: $\mathrm{OR}=5.75,95 \%$ $\mathrm{CI}=2.22-14.92, P=0.0003$; albuminuria: $\mathrm{OR}=15.42,95 \%$
$\mathrm{CI}=5.39-44.10, P<0.00001$; hand-foot syndrome: $\mathrm{OR}=2.09$, $95 \% \mathrm{CI}=1.26-3.48, P=0.004)$, whereas analyses of leukopenia, thrombocytopenia, diarrhea, nausea and vomiting, neutropenia, oral mucositis, weak, hemoglobin reduction,

Table 2 Information of apatinib targeted therapy combined with CT

\begin{tabular}{|c|c|c|c|c|c|}
\hline \multirow[t]{2}{*}{ Included studies } & \multicolumn{2}{|l|}{ Therapeutic regimen } & \multirow[t]{2}{*}{ Line } & \multirow{2}{*}{$\begin{array}{l}\text { Enrollment } \\
\text { period }\end{array}$} & \multirow[t]{2}{*}{ Dosage of apatinib } \\
\hline & $\operatorname{Exp}$ & Con & & & \\
\hline Dong et al, ${ }^{19} 2018$ & Oxaliplatin+apatinib & Oxaliplatin & ND & $2013.1-2013.12$ & 850 mg/time, 3 times $/ \mathrm{d}$ \\
\hline $\mathrm{Du},{ }^{20} 2017$ & FBC+apatinib & Fluorouracil & ND & $2015.3-2017.4$ & 500 mg/time, I time/d \\
\hline Duan et al, 2017 & FOLFOX+apatinib & FOLFOX & ND & $2015.1-2016.12$ & $850 \mathrm{mg} /$ time, I time $/ \mathrm{d}$ \\
\hline Fan et al, ${ }^{22} 2017$ & S-I+apatinib & S-I & 1 & $20 \mid 5.1-2016.1$ & $500 \mathrm{mg} /$ time, I time $/ \mathrm{d}$ \\
\hline Gao et al, ${ }^{23} 2017$ & S-I+apatinib & S-I & ND & $2015.6-2016.6$ & $500-850 \mathrm{mg} /$ time, I time $/ \mathrm{d}$ \\
\hline Hu et al, ${ }^{24} 2016$ & S-I+apatinib & S-I & I & $2015.1-2016.8$ & $800-850 \mathrm{mg} /$ time, I time $/ \mathrm{d}$ \\
\hline Jing, ${ }^{25} 2016$ & S-I+apatinib & S-I & 1 & $2014.11-2015.12$ & $500 \mathrm{mg} /$ time, I time $/ \mathrm{d}$ \\
\hline Li et al, ${ }^{26} 2018$ & FOLFOX+apatinib & FOLFOX & $\geq \mathrm{III}$ & $2014.12-2016.12$ & $250-850 \mathrm{mg} /$ time, I time $/ \mathrm{d}$ \\
\hline $\mathrm{Li}$ and $\mathrm{Li}^{27} 20 \mathrm{I} 7$ & Oxaliplatin+S-I+apatinib & Oxaliplatin+S-I & $\geq I I I$ & $20 \mid 5.2-2016.2$ & $850 \mathrm{mg} /$ time, I time $/ \mathrm{d}$ \\
\hline $\mathrm{Li}^{28} 20 \mathrm{I7}$ & FBC+apatinib & Fluorouracil & ND & ND & $250 \mathrm{mg} /$ time, I time $/ \mathrm{d}$ \\
\hline Qian and $\mathrm{Ge},{ }^{29} 2017$ & $\begin{array}{l}\text { Taxanes/platinum/ } \\
\text { fluorouracil+apatinib }\end{array}$ & $\begin{array}{l}\text { Taxanes/platinum/ } \\
\text { fluorouracil }\end{array}$ & ND & $2015.6-2017.6$ & $500 \mathrm{mg} /$ time, I time $/ \mathrm{d}$ \\
\hline Sheng et $\mathrm{al},{ }^{30} 2017$ & S-I+apatinib & S-I & ॥ & ND & $250 \mathrm{mg} /$ time, 2 times $/ \mathrm{d}$ \\
\hline Wang et al, ${ }^{31} 2016$ & S-I+apatinib & S-I & ND & $2015.2-2016.6$ & $850 \mathrm{mg} /$ time, I time $/ \mathrm{d}$ \\
\hline Wen et al, 2017 & Oxaliplatin+S-I+apatinib & Oxaliplatin+S-I & ND & $20|4.1-20| 6.4$ & $850 \mathrm{mg} /$ time, I time $/ \mathrm{d}$ \\
\hline Wu et al, ${ }^{33} 2017$ & S-I+apatinib & S-I & ॥ & $2015.12-2017.2$ & $500 \mathrm{mg} /$ time, I time $/ \mathrm{d}$ \\
\hline Yan et al, ${ }^{34} 2017$ & Oxaliplatin+S-I+apatinib & Oxaliplatin+S-I & $\geq I I I$ & $2015.3-2017.3$ & $850 \mathrm{mg} /$ time, I time $/ \mathrm{d}$ \\
\hline Zhan, ${ }^{35} 2017$ & FOLFIRI+apatinib & FOLFIRI & ND & $2011.5-2013.5$ & $425-850 \mathrm{mg} / \mathrm{d}, \mathrm{I}$ time/d \\
\hline Zhou et al, ${ }^{36} 2018$ & S-I+apatinib & S-I & $\geq I I$ & $2015.7-2016.10$ & $850 \mathrm{mg} /$ time, I time $/ \mathrm{d}$ \\
\hline Zhu et $a \mid,{ }^{37} 2016$ & $\begin{array}{l}\text { Taxanes/irinotecan/ } \\
\text { fluorouracil+apatinib }\end{array}$ & $\begin{array}{l}\text { Taxanes/irinotecan/ } \\
\text { fluorouracil }\end{array}$ & ND & $20 \mid 4.7-2016.7$ & $500 \mathrm{mg} /$ time, I time $/ \mathrm{d}$ \\
\hline
\end{tabular}

Notes: Con, control group (CT alone group); Exp, experimental group (apatinib targeted therapy plus CT).

Abbreviations: CT, chemotherapy; FBC, fluorouracil-based chemotherapy; FOLFIRI, calcium folinate+irinotecan+5-fluorouracil; FOLFOX, oxaliplatin+calcium folinate+ 5 -fluorouracil; ND, not determined; S-I, gimeracil and oteracil porassium capsules. 
B

A

\begin{tabular}{|c|c|c|c|c|c|c|c|}
\hline & 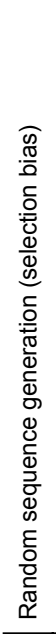 & 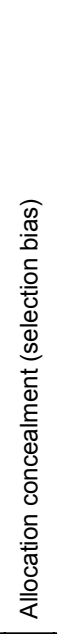 & 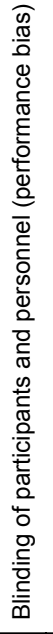 & $\overline{\mathbf{\omega}}$ & 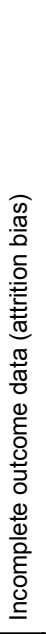 & 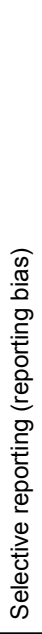 & 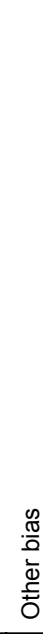 \\
\hline Dong et al, ${ }^{19} 2018$ & + & + & $?$ & $?$ & + & + & $?$ \\
\hline Duan et al, ${ }^{, 1} 2017$ & + & + & $?$ & $?$ & $\oplus$ & $\oplus$ & + \\
\hline $\mathrm{Du}_{, 2}^{20} 2017$ & $\theta$ & $\theta$ & $?$ & $?$ & + & + & $?$ \\
\hline Fan et al, ${ }^{22} 2017$ & + & + & $?$ & $?$ & + & $\oplus$ & + \\
\hline Gao et al, ${ }^{23} 2017$ & $\odot$ & + & $?$ & $?$ & + & $\odot$ & $?$ \\
\hline Hu et al, ${ }^{24} 2016$ & + & + & $?$ & $?$ & + & + & $?$ \\
\hline Jing, ${ }^{25} 2016$ & + & + & $?$ & $?$ & + & + & + \\
\hline Li et al, ${ }^{26} 2018$ & $\theta$ & $\theta$ & $?$ & $?$ & $\oplus$ & $?$ & + \\
\hline $\mathrm{Li}$ and $\mathrm{Li},{ }^{27} 2017$ & $\theta$ & $\theta$ & $?$ & $?$ & + & $?$ & + \\
\hline $\mathrm{Li}^{28} 2017$ & + & + & $?$ & $?$ & $\oplus$ & + & $?$ \\
\hline Qian and $\mathrm{Ge},{ }^{29} 2017$ & $\theta$ & $\theta$ & $?$ & $?$ & + & $\odot$ & + \\
\hline Sheng et al, ${ }^{30} 2017$ & + & + & $?$ & $?$ & + & + & $?$ \\
\hline Wang et al, ${ }^{31} 2016$ & + & + & $?$ & $?$ & + & $?$ & $?$ \\
\hline Wen et al, ${ }^{32} 2017$ & + & + & $?$ & $?$ & + & $\oplus$ & $?$ \\
\hline Wu et al, ${ }^{33} 2017$ & + & + & $?$ & $?$ & + & + & + \\
\hline Yan et al, ${ }^{34} 2017$ & + & + & $?$ & $?$ & $\oplus$ & + & + \\
\hline Zhan, ${ }^{35} 2017$ & $\oplus$ & + & $?$ & $?$ & + & $\odot$ & + \\
\hline Zhou et al, ${ }^{36} 2018$ & + & + & $?$ & $?$ & + & + & $?$ \\
\hline Zhu et al, ${ }^{37} 2016$ & $\theta$ & $\theta$ & $?$ & $?$ & + & + & + \\
\hline
\end{tabular}

Random sequence generation (selection bias)

Allocation concealment (selection bias)

Blinding of participants and personnel (performance bias)

Blinding of outcome assessment (detection bias)

Incomplete outcome data (attrition bias)

Selective reporting (reporting bias)

Other bias

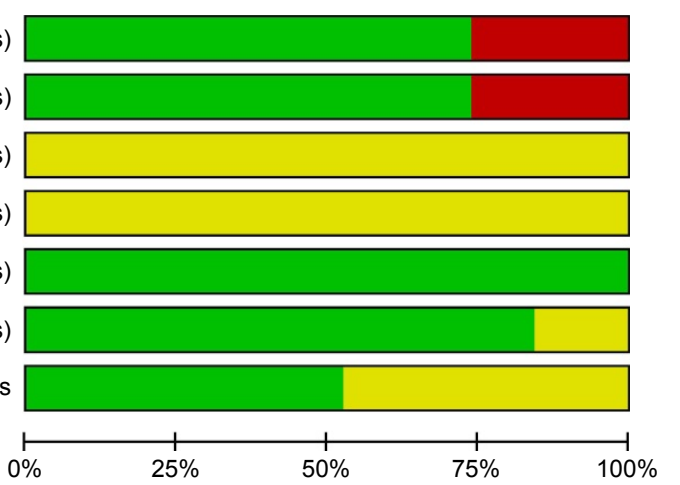

$0 \%$

Low risk of bias $\square$ Unclear risk of bias

High risk of bias

Figure 2 (A) Risk of bias summary: review of the authors' judgments about each risk of bias item for the included studies, (B) risk of bias graph: review of the authors' judgments about each risk of bias item presented as percentages across all included studies.

Note: Each color represents a different level of bias: red for high risk, green for low risk, and yellow for unclear risk of bias. 


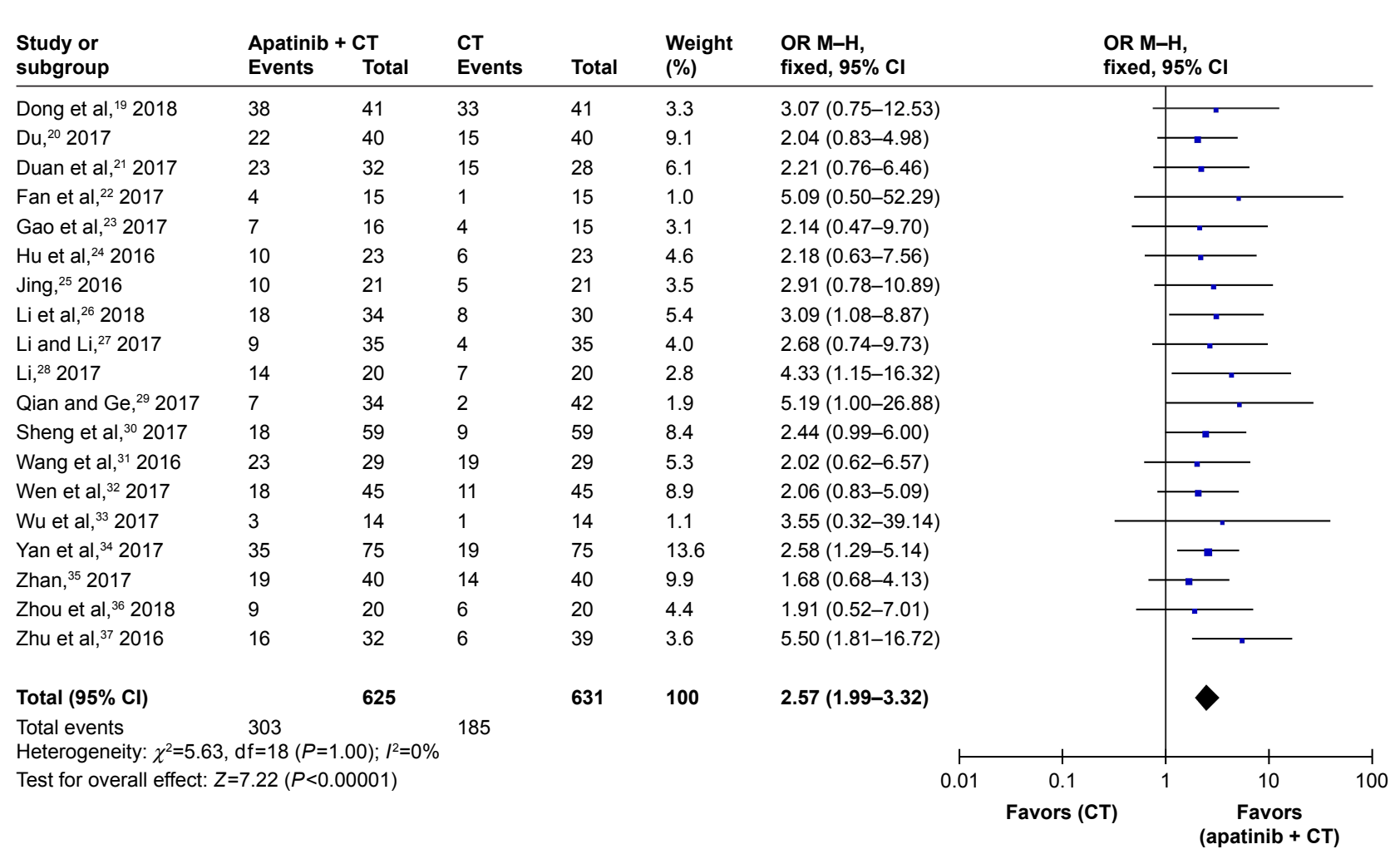

Figure 3 Forest plot of the comparison of the ORR between the experimental and control group.

Notes: Control group, CT alone group; experimental group, apatinib targeted therapy plus CT. The fixed effects meta-analysis model (Mantel-Haenszel method) was used. Abbreviations: $\mathrm{Cl}$, confidence interval; $\mathrm{CT}$, chemotherapy; OR, odds ratio; ORR, overall response rate.

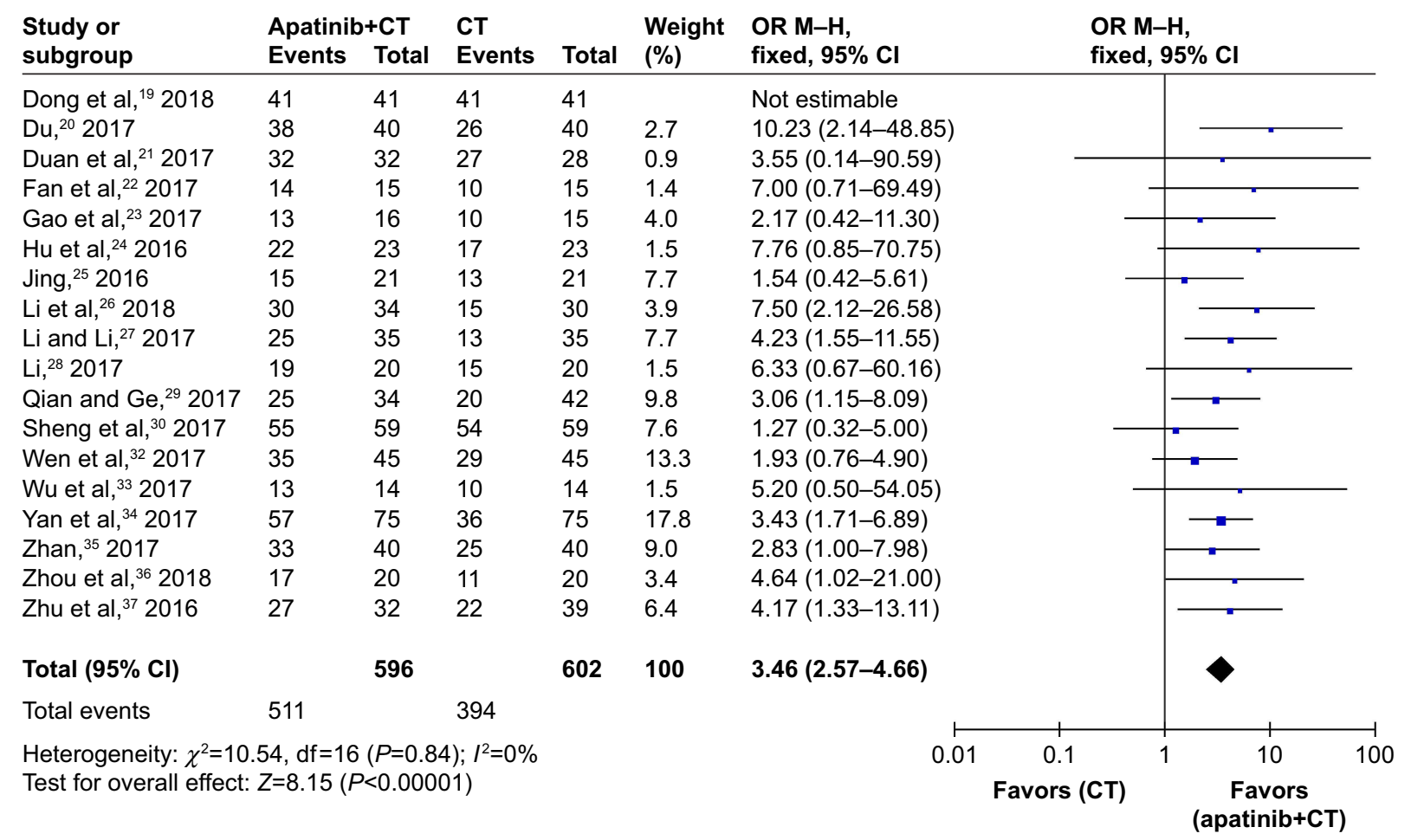

Figure 4 Forest plot of the comparison of the DCR between the experimental and control group.

Notes: Control group, CT alone group; experimental group, apatinib targeted therapy plus CT. The fixed effects meta-analysis model (Mantel-Haenszel method) was used. Abbreviations: $\mathrm{Cl}$, confidence interval; CT, chemotherapy; DCR, disease control rate; OR, odds ratio. 
Table 3 Comparison of CR, PR, SD, PD, ORR, and DCR between the experimental and control groups

\begin{tabular}{|c|c|c|c|c|c|c|c|c|}
\hline \multirow[t]{2}{*}{ Parameter } & \multirow{2}{*}{$\begin{array}{l}\text { Exp } \\
\text { Number of } \\
\text { patients (n) }\end{array}$} & \multirow{2}{*}{$\begin{array}{l}\text { Con } \\
\text { Number of } \\
\text { patients (n) }\end{array}$} & \multirow[t]{2}{*}{ Analysis method } & \multicolumn{2}{|c|}{ Heterogeneity } & \multirow[t]{2}{*}{ OR } & \multirow[t]{2}{*}{$95 \% \mathrm{Cl}$} & \multirow[t]{2}{*}{$P$-value } \\
\hline & & & & $I^{2}(\%)$ & $P$-value & & & \\
\hline $\mathrm{CR}$ & 625 & 631 & Fixed & 0 & 0.96 & 1.85 & $1.04-3.28$ & 0.04 \\
\hline PR & 625 & 631 & Fixed & 0 & 0.89 & 2.19 & $1.71-2.80$ & $<0.0000 \mathrm{I}$ \\
\hline SD & 596 & 602 & Fixed & 0 & 0.59 & 1.09 & $0.86-1.39$ & 0.48 \\
\hline PD & 596 & 602 & Fixed & 0 & 0.94 & 0.33 & $0.25-0.44$ & $<0.00001$ \\
\hline ORR & 625 & 631 & Fixed & 0 & 1.00 & 2.57 & $1.99-3.32$ & $<0.00001$ \\
\hline DCR & 596 & 602 & Fixed & 0 & 0.84 & 3.46 & $2.57-4.66$ & $<0.00001$ \\
\hline
\end{tabular}

Notes: Con, control group (CT alone group); Exp, experimental group (apatinib targeted therapy plus CT).

Abbreviations: $C R$, complete response rates; $C T$, chemotherapy; DCR, disease control rate; OR, odds ratio; ORR, overall response rate; PD, progressive disease rates; $\mathrm{PR}$, partial response rates; $\mathrm{SD}$, stable disease rates.

and myelosuppression did not reveal significant differences (leukopenia: $\mathrm{OR}=1.73,95 \% \mathrm{CI}=0.96-3.10, P=0.07$; thrombocytopenia: $\mathrm{OR}=1.31,95 \% \mathrm{CI}=0.79-2.18, P=0.29$; diarrhea: $\mathrm{OR}=0.63,95 \% \mathrm{CI}=0.36-1.10, P=0.10$; nausea and vomiting: $\mathrm{OR}=1.02,95 \% \mathrm{CI}=0.71-1.46, P=0.92$; neutropenia: $\mathrm{OR}=1.33,95 \% \mathrm{CI}=0.68-2.59, P=0.40$; oral mucositis: $\mathrm{OR}=1.19,95 \% \mathrm{CI}=0.80-1.77, P=0.40$; weak: $\mathrm{OR}=1.09,95 \%$ $\mathrm{CI}=0.70-1.70, P=0.70$; hemoglobin reduction: $\mathrm{OR}=2.13$, 95\% CI=0.69-6.59, $P=0.19$; myelosuppression: $\mathrm{OR}=0.96$, $95 \% \mathrm{CI}=0.57-1.63, P=0.89)$.

\section{Publication bias}

The funnel plots drawn for the studies of the primary outcomes (CR, PR, SD, PD, ORR, DCR, and AEs) were approximately symmetrical, which indicated the generally controlled publication bias and reliability of our primary conclusions (Figures 6 and $\underline{\mathrm{S} 3}$ and $\underline{\mathrm{S} 4}$ ).

\section{Sensitivity analysis}

We conducted subgroup analyses to explore the sources of heterogeneity in the ORR and DCR with respect to therapeutic regimens, apatinib dosages, sample sizes, and types of involved studies. As presented in Table 5, our analysis results revealed that no significant differences were found between the different therapeutic regimens, apatinib dosages, sample sizes, and types of studies.

\section{Discussion}

In recent years, with the development of tumor molecular biology and epigenetics, increasing numbers of targeted

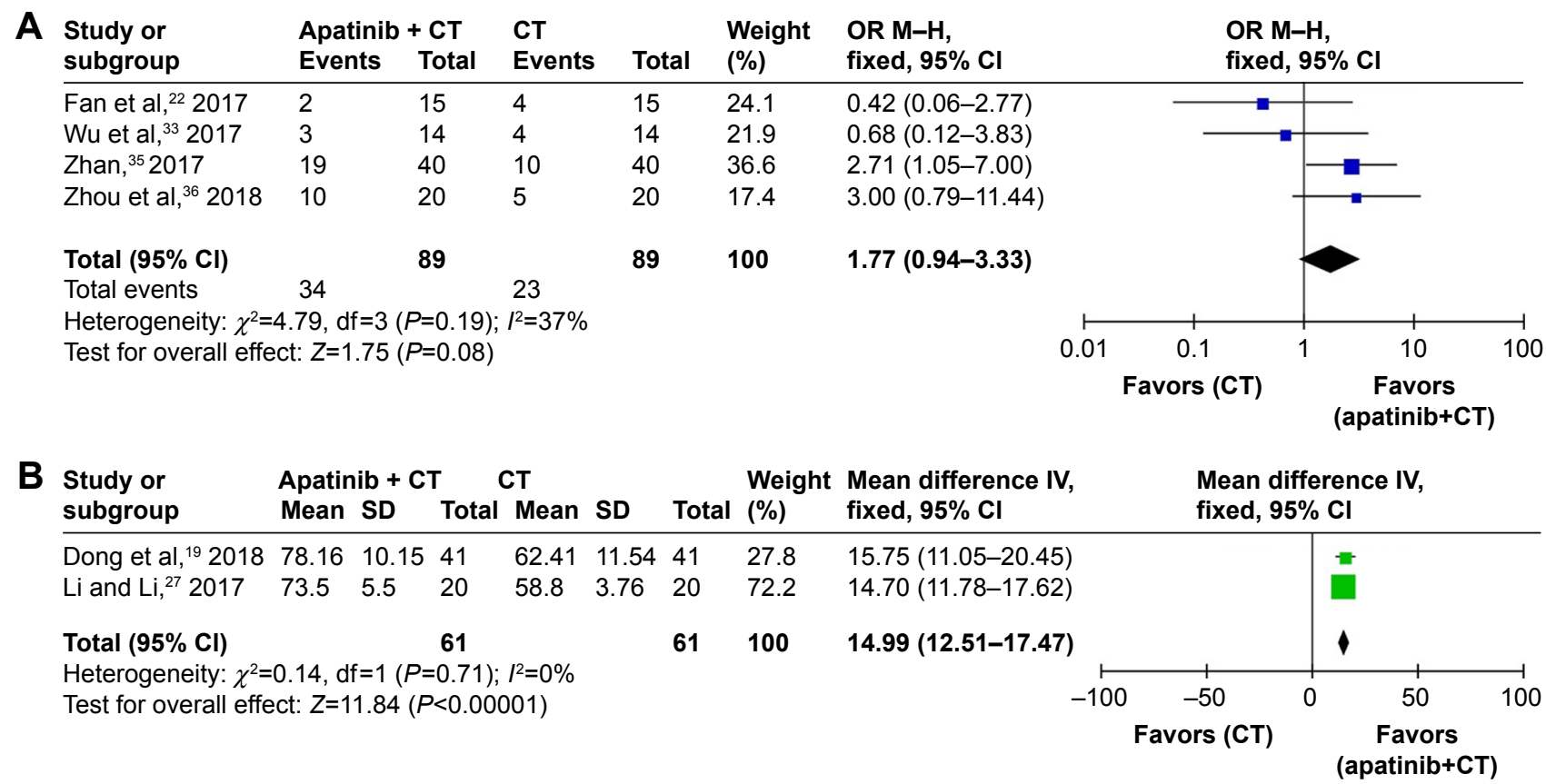

Figure 5 Funnel plot of percentages of the ORR (A) and DCR (B) between the experimental and control groups. Abbreviations: $\mathrm{Cl}$, confidence interval; $\mathrm{CT}$, chemotherapy; $\mathrm{DCR}$, disease control rate; ORR, overall response rate. 
Table 4 Comparison of AEs between the experimental and control groups

\begin{tabular}{|c|c|c|c|c|c|c|c|c|}
\hline \multirow[t]{2}{*}{ AEs } & \multirow{2}{*}{$\begin{array}{l}\text { Exp } \\
\text { Number of } \\
\text { patients (n) }\end{array}$} & \multirow{2}{*}{$\begin{array}{l}\text { Con } \\
\text { Number of } \\
\text { patients (n) }\end{array}$} & \multirow[t]{2}{*}{ Analysis method } & \multicolumn{2}{|c|}{ Heterogeneity } & \multirow[t]{2}{*}{ OR } & \multirow[t]{2}{*}{$95 \% \mathrm{Cl}$} & \multirow[t]{2}{*}{$P$-value } \\
\hline & & & & $I^{2}(\%)$ & $P$-value & & & \\
\hline Leukopenia & 123 & 118 & Fixed & 0 & 0.67 & 1.73 & $0.96-3.10$ & 0.07 \\
\hline Thrombocytopenia & 244 & 239 & Fixed & 0 & 0.91 & 1.31 & $0.79-2.18$ & 0.29 \\
\hline Diarrhea & 252 & 251 & Fixed & 0 & 0.75 & 0.63 & $0.36-1.10$ & 0.10 \\
\hline Nausea, vomiting & 348 & 355 & Fixed & 25 & 0.21 & 1.02 & $0.7 I-I .46$ & 0.92 \\
\hline Hypertension & 392 & 395 & Random & 60 & 0.005 & 5.75 & $2.22-14.92$ & 0.0003 \\
\hline Neutropenia & 120 & 120 & Fixed & 0 & 0.83 & 1.33 & $0.68-2.59$ & 0.40 \\
\hline Albuminuria & 175 & 182 & Fixed & 0 & 0.62 & 15.42 & $5.39-44.10$ & $<0.00001$ \\
\hline Oral mucositis & 274 & 281 & Fixed & 33 & 0.16 & 1.19 & $0.80-1.77$ & 0.40 \\
\hline Hand-foot syndrome & 262 & 257 & Fixed & 0 & 0.57 & 2.09 & $1.26-3.48$ & 0.004 \\
\hline Weak & 209 & 208 & Fixed & 0 & 0.58 & 1.09 & $0.70-1.70$ & 0.70 \\
\hline Hemoglobin reduction & 77 & 72 & Random & 55 & 0.08 & 2.13 & $0.69-6.59$ & 0.19 \\
\hline Myelosuppression & 129 & 137 & Fixed & 0 & 0.79 & 0.96 & $0.57-1.63$ & 0.89 \\
\hline
\end{tabular}

Notes: Con, control group (CT alone group); Exp, experimental group (apatinib targeted therapy plus CT).

Abbreviations: $A E$, adverse event; $C T$, chemotherapy; OR, odds ratio.

agents, such as gefitinib, erlotinib, apatinib, etc, have been used to improve treatment effects for patients with malignancies. ${ }^{40-43}$ As components of the important signaling pathway of cancer angiogenesis, VEGF and VEGFR are closely related to cancer invasiveness. Researchers have confirmed that the expressions of VEGF and VEGFR are associated with poor prognosis in GC. ${ }^{44,45}$ Upon binding to its receptors, the activated VEGF family promotes the proliferation of vascular cells for the development of new blood vessels in tumor tissues and then ensures oxygen and nutrient supplies and causes tumor growth and metastasis. ${ }^{14,46}$ Therefore, anti-VEGFR target drugs are considered promising prospects for the treatment of advanced GC.

The VEGFR family includes VEGFR-1, VEGFR-2, and VEGFR-3. ${ }^{16}$ Among these receptors, VEGFR2 plays an essential role in VEGF-mediated tumor angiogenesis. ${ }^{14,17}$

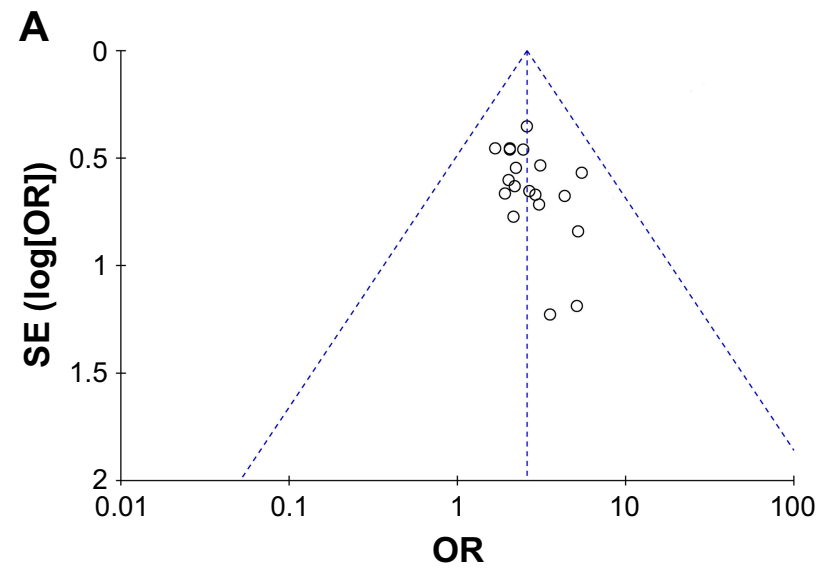

When it associates with VEGF, the dimerization of VEGFR2 causes the autophosphorylation of intracellular tyrosine kinase domains, which leads to the activation of the PLC$\gamma$-Raf kinase-MEK-MAP kinase pathway, which in turn enhances endothelial cell proliferation. ${ }^{14,15}$ Apatinib is a new inhibitor of VEGFR-2 tyrosine kinase that targets the intracellular ATP binding site of the receptor. ${ }^{17}$ Several studies have reported that the addition of apatinib can be beneficial for patients with advanced GC. ${ }^{42,43}$ Although there are statistical analyses of published clinical trials, the exact therapeutic effects have not been systematically evaluated and demonstrated due to sample size variability among these trials. Additionally, the different applied protocols in the different clinical trials may have led to different therapeutic effects. In the present research, we performed an extensive online search followed by rigorous contrasting and combining data

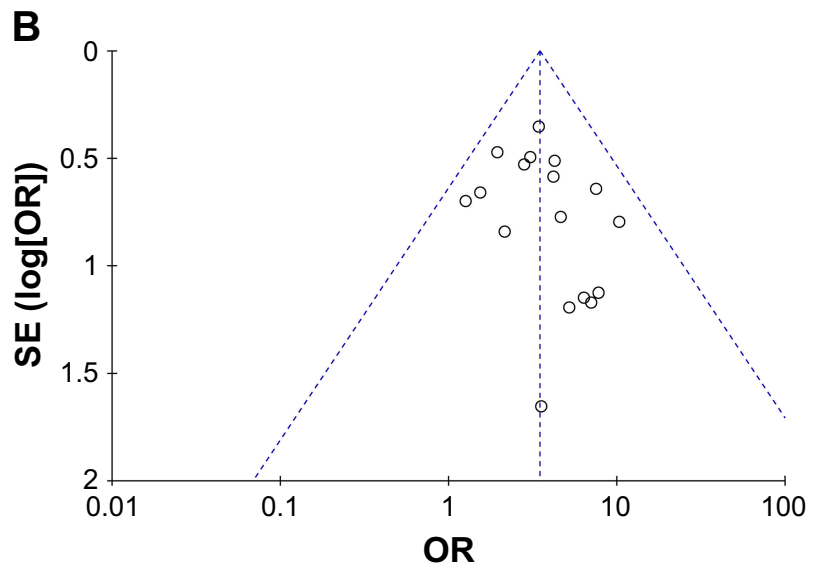

Figure 6 Forest plot of the comparison of the QIR (A) and KPS (B) between the experimental and control groups.

Notes: Control group, CT alone group; experimental group, apatinib targeted therapy plus CT. The fixed effects meta-analysis model (Mantel-Haenszel method) was used. Abbreviations: Cl, confidence interval; CT, chemotherapy; KPS, Karnofsky performance score; QIR, quality of life improved rate. 
Table 5 Subgroup analyses of ORR and DCR between the experimental and control groups

\begin{tabular}{|c|c|c|c|c|c|c|c|c|c|}
\hline \multirow[t]{2}{*}{ Parameter } & \multirow[t]{2}{*}{ Factors at study level } & \multirow{2}{*}{$\begin{array}{l}\text { Exp } \\
\text { Number of } \\
\text { patients (n) }\end{array}$} & \multirow{2}{*}{$\begin{array}{l}\text { Con } \\
\text { Number of } \\
\text { patients (n) }\end{array}$} & \multirow[t]{2}{*}{ Analysis method } & \multicolumn{2}{|c|}{ Heterogeneity } & \multirow[t]{2}{*}{ OR } & \multirow[t]{2}{*}{$95 \% \mathrm{Cl}$} & \multirow[t]{2}{*}{$P$-value } \\
\hline & & & & & $I^{2}(\%)$ & $P$-value & & & \\
\hline \multirow[t]{14}{*}{ ORR } & Therapeutic regimen & & & & & & & & \\
\hline & Apatinib+FOLFOX & 66 & 58 & Fixed & 0 & 0.66 & 2.63 & $1.24-5.56$ & 0.01 \\
\hline & Apatinib+S-I+oxaliplatin & 155 & 155 & Fixed & 0 & 0.91 & 2.42 & $1.46-4.01$ & 0.0006 \\
\hline & Apatinib+FBC & 60 & 60 & Fixed & 0 & 0.35 & 2.58 & I.24-5.39 & 0.01 \\
\hline & Apatinib+S-I & 197 & 196 & Fixed & 0 & 1.00 & 2.40 & $1.5 \mathrm{I}-3.82$ & 0.0002 \\
\hline & Dosage of apatinib & & & & & & & & \\
\hline & $850 \mathrm{mg} / \mathrm{d}$ & 236 & 232 & Fixed & 0 & 1.00 & 2.29 & $1.53-3.43$ & $<0.0001$ \\
\hline & $500 \mathrm{mg} / \mathrm{d}$ & 215 & 230 & Fixed & 0 & 0.84 & 3.07 & $1.94-4.88$ & $<0.00001$ \\
\hline & Study sample size & & & & & & & & \\
\hline & $>50$ & 496 & 503 & Fixed & 0 & 0.96 & 2.52 & $1.89-3.37$ & $<0.00001$ \\
\hline & $<50$ & 129 & 128 & Fixed & 0 & 0.97 & 2.75 & $1.57-4.79$ & 0.0004 \\
\hline & Type of control trials & & & & & & & & \\
\hline & RCT & 450 & 445 & Fixed & 0 & 1.00 & 2.38 & $1.77-3.22$ & $<0.00001$ \\
\hline & Non-RCT & 175 & 186 & Fixed & 0 & 0.68 & 3.16 & $1.92-5.20$ & $<0.00001$ \\
\hline \multirow[t]{14}{*}{ DCR } & Therapeutic regimen & & & & & & & & \\
\hline & Apatinib+FOLFOX & 66 & 58 & Fixed & 0 & 0.67 & 6.74 & $2.07-21.95$ & 0.002 \\
\hline & Apatinib+S-I+oxaliplatin & 155 & 155 & Fixed & 0 & 0.49 & 3.07 & $1.89-5.00$ & $<0.00001$ \\
\hline & Apatinib+FBC & 60 & 60 & Fixed & 0 & 0.73 & 8.80 & $2.45-31.70$ & 0.0009 \\
\hline & Apatinib $+S-I$ & 168 & 167 & Fixed & 0 & 0.63 & 2.78 & $1.5 \mid-5.10$ & 0.0010 \\
\hline & Dosage of apatinib & & & & & & & & \\
\hline & $850 \mathrm{mg} / \mathrm{d}$ & 207 & 203 & Fixed & 0 & 0.79 & 3.21 & $2.03-5.07$ & $<0.00001$ \\
\hline & $500 \mathrm{mg} / \mathrm{d}$ & 215 & 230 & Fixed & 0 & 0.43 & 3.32 & $2.02-5.48$ & $<0.00001$ \\
\hline & Study sample size & & & & & & & & \\
\hline & $>50$ & 467 & 474 & Fixed & 0 & 0.61 & 3.43 & $2.45-4.79$ & $<0.00001$ \\
\hline & $<50$ & 129 & 128 & Fixed & 0 & 0.78 & 3.58 & $1.86-6.90$ & 0.0001 \\
\hline & Type of control trials & & & & & & & & \\
\hline & RCT & 421 & 416 & Fixed & 0 & 0.89 & 2.88 & $1.98-4.18$ & $<0.00001$ \\
\hline & Non-RCT & 175 & 186 & Fixed & 0 & 0.68 & 4.79 & $2.89-7.92$ & $<0.00001$ \\
\hline
\end{tabular}

Notes: Con, control group (CT alone group); Exp, experimental group (apatinib targeted therapy plus CT).

Abbreviations: $\mathrm{Cl}$, confidence interval; CT, chemotherapy; DCR, disease control rate; FBC, fluorouracil-based chemotherapy; FOLFOX, oxaliplatin+calcium folinate+5fluorouracil; OR, odds ratio; ORR, overall response rate; QIR, quality of life improved rate; RCT, randomized controlled trial; S-I, gimeracil and oteracil porassium capsules.

analyses in terms of categorization to provide a clear and systematical conclusion.

Our meta-analysis revealed that apatinib targeted therapy combined with CT is associated with a favorable efficacy compared with $\mathrm{CT}$ alone. Compared with the patients who were treated with $\mathrm{CT}$ alone, the patients who were treated with combined therapy exhibited markedly increased CR, PR, ORR, and DCR $(P<0.05)$. The patients' QOL was also evaluated in this analysis, and the QOL was significantly improved after combined therapy. These results indicated that apatinib targeted therapy increased the curative effect of $\mathrm{CT}$ by inhibiting tumor angiogenesis and thereby improving the patients' life qualities.

Safety is the top priority of clinical treatment, and it is also the key factor for the development of apatinib targeted therapy. Regarding AEs and severe toxicities, our analysis revealed that there were no significant differences in most of the $\mathrm{AE}$ indicators between the 2 groups. The group receiving CT plus apatinib targeted therapy had higher rates of hypertension, proteinuria, and hand-foot syndrome, which are usually controllable events and do not require permanent discontinuation of therapy.

Some factors may influence the therapeutic effects of apatinib targeted therapy. In our subgroup analysis, no differences were found between the different therapeutic regimens, apatinib dosages, sample sizes, and types of studies. However, currently published studies that have probed the influences of these factors on the curative effect of apatinib targeted therapy are still insufficient; thus, these issues should be further researched and explored. Furthermore, the determination of the optimal therapeutic strategy will be valuable for GC treatment. Recently, many novel treatment strategies, such as targeted therapy and immunotherapy, have been developed for the treatment of malignancies. Several 
studies have found that combined treatment with targeted therapy and immunotherapy for malignant tumors has better therapeutic effects than single therapy. ${ }^{47,48}$ Therefore, the combination of targeted therapy with an immunotherapy, such as chimeric antigen receptor-modified $\mathrm{T}$ cells, T-cell receptor-modified $\mathrm{T}$ cells, etc, ${ }^{49,50}$ may be the new direction for the future development of advanced GC treatment.

There are some limitations in our analysis. First, the number of GC patients included in this study is not sufficiently large, and the follow-up time was short. Apart from that, the different trials evaluated the treatment efficacy using different outcomes, so it was difficult to summarize the results on the same scale, which led to shrunken statistical sample sizes. Third, our data were partly extracted from published papers rather than original patient records, which mean that we were not able to avoid analytical bias based on the information presented in the articles. Due to the above limitations, future studies and generated data will be valuable to further verify the safety and efficacy of apatinib targeted therapy.

\section{Conclusion}

In summary, our study confirmed that apatinib targeted therapy combined with CT is an effective treatment for advanced GC patients. Apatinib targeted therapy markedly enhances the treatment efficacy of CT for advanced GC. However, this combined treatment could lead to greater rates of hypertension, albuminuria, and hand-foot syndrome. Therefore, the benefits and risks should be considered before treatment.

\section{Author contributions}

All authors contributed toward data analysis, drafting, and critically revising the paper and agree to be accountable for all aspects of the work.

\section{Disclosure}

The authors report no conflicts of interest in this work.

\section{References}

1. Mu Y, Zhou CH, Chen SF, et al. Effectiveness and safety of chemotherapy combined with cytokine-induced killer cell/dendritic cellcytokine-induced killer cell therapy for treatment of gastric cancer in China: a systematic review and meta-analysis. Cytotherapy. 2016;18(9): 1162-1177.

2. Xie $\mathrm{S}$, Zhang $\mathrm{H}$, Wang $\mathrm{X}, \mathrm{Ge} \mathrm{Q}, \mathrm{Hu}$ J. The relative efficacy and safety of targeted agents used in combination with chemotherapy in treating patients with untreated advanced gastric cancer: a network meta-analysis. Oncotarget. 2017;8(16):26959-26968.

3. Chen W, Zheng R, Zeng H, Zhang S, He J. Annual report on status of cancer in China, 2011. Chin J Cancer Res. 2015;27(1):2-12.

4. Kalnina Z, Meistere I, Kikuste I, Tolmanis I, Zayakin P, Line A. Emerging blood-based biomarkers for detection of gastric cancer. World $J$ Gastroenterol. 2015;21(41):11636-11653.
5. Digklia A, Wagner AD. Advanced gastric cancer: current treatment landscape and future perspectives. World J Gastroenterol. 2016;22(8): 2403-2414.

6. Wagner AD, Syn NL, Moehler M, et al. Chemotherapy for advanced gastric cancer. Cochrane Database Syst Rev. 2017;8:CD004064.

7. Mellman I, Coukos G, Dranoff G. Cancer immunotherapy comes of age. Nature. 2011;480(7378):480-489.

8. Satoh T, Lee KH, Rha SY, et al. Randomized phase II trial of nimotuzumab plus irinotecan versus irinotecan alone as second-line therapy for patients with advanced gastric cancer. Gastric Cancer. 2015;18(4):824-832.

9. Bang YJ, Van Cutsem E, Feyereislova A, et al. Trastuzumab in combination with chemotherapy versus chemotherapy alone for treatment of HER2-positive advanced gastric or gastro-oesophageal junction cancer (ToGA): a phase 3, open-label, randomised controlled trial. Lancet. 2010;376(9742):687-697.

10. Luo HQ, Han L, Jiang Y. Meta-analysis of six randomized control trials of chemotherapy plus anti-HER monoclonal antibody for advanced gastric and gastroesophageal cancer. Asian Pac J Cancer Prev. 2014;15(13): 5343-5348.

11. Wilke H, Muro K, Van Cutsem E, et al. Ramucirumab plus paclitaxel versus placebo plus paclitaxel in patients with previously treated advanced gastric or gastro-oesophageal junction adenocarcinoma (RAINBOW): a double-blind, randomised phase 3 trial. Lancet Oncol. 2014;15(11):1224-1235.

12. Roviello G, Polom K, Roviello F, et al. Targeting VEGFR-2 in metastatic gastric cancer: results from a literature-based meta-analysis. Cancer Invest. 2017;35(3):187-194.

13. Botrel TEA, Clark LGO, Paladini L, Clark OAC. Efficacy and safety of bevacizumab plus chemotherapy compared to chemotherapy alone in previously untreated advanced or metastatic colorectal cancer: a systematic review and meta-analysis. BMC Cancer. 2016;16:677.

14. Zhao J, Zhang X, Gong C, Zhang J. Targeted therapy with apatinib in a patient with relapsed small cell lung cancer: a case report and literature review. Medicine. 2017;96(50):e9259.

15. Fontanella C, Ongaro E, Bolzonello S, Guardascione M, Fasola G, Aprile G. Clinical advances in the development of novel VEGFR2 inhibitors. Ann Transl Med. 2014;2(12):123.

16. Ferrara N, Gerber HP, LeCouter J. The biology of VEGF and its receptors. Nat Med. 2003;9(6):669-676.

17. Roviello G, Ravelli A, Polom K, et al. Apatinib: a novel receptor tyrosine kinase inhibitor for the treatment of gastric cancer. Cancer Lett. 2016;372(2):187-191.

18. Zhang H. Apatinib for molecular targeted therapy in tumor. Drug Des Devel Ther. 2015;9:6075-6081.

19. Dong GC, Li LX, Wang GX. Effect of oxaliplatin and apatinib combined therapy on survival and platelets of patients with advanced gastric cancer. Contemp Med. 2018;24(4):77-79.

20. Du W. Effect analysis of apatinib combined with chemotherapy in the treatment of advanced gastric cancer. J Clin Med Lit. 2017;4(49): 9661-9662.

21. Duan HR, Song Y, Jiang XY. Observation of curative effect of apatinib combined with FOLFOX chemotherapy regimen in the treatment of advanced gastric cancer. China Pract Med. 2017;12(28):155-157.

22. Fan XX, Lv HF, Chen BB, et al. Clinical study of Apatinib combined with S-1 in the treatment of patients with advanced gastric cancer and review of literature. Chin J Front Med Sci. 2017;9(2):63-67.

23. Gao JP, Han T, Piao Y, et al. Apatinib combined tegafur in treatment for elderly or emaciated patients with advanced gastric cancer. Clin J Med Officers. 2017;45(1):9-12.

24. Hu SS, Xiang M, Lu P, Zhang WZ. Clinical effect analysis of apatinib plus $\mathrm{S}-1$ in the treatment of elderly patients with advanced gastric cancer. Clin Med. 2016;36:33.

25. Jing XH. Effect of apatinib combined with S-1 as first-line therapy on elderly patients with advanced gastric cancer. Chin J Pract Med. 2016;43(10):37-39. 
26. Li S, Zhong YJ, Teng Y, Zhu LJ. Clinical efficacy and prognosis of apatinib combined with chemotherapy in the treatment of advanced gastric cancer. Chin J Clin Oncol Rehab. 2018;25(1):44-46.

27. Li P, Li CH. Comparative analysis of the efficacy and safety of apatinib tablet combined with oxaliplatin Injection and tegafur capsule in the treatment of advanced gastric cancer. Hebei Med. 2017;23(11): 1923-1926.

28. Li Q. Clinical effect analysis of apatinib in the treatment of patients with chemotherapy drug resistant advanced gastric cancer. $J$ Imaging Res Med Appl. 2017;1(4):226-227.

29. Qian XY, Ge W. Apatinib combined with chemotherapy for advanced gastric cancer. J Chin Pract Diagn Ther. 2017;31(12):1222-1224.

30. Sheng HM, Wu C, Deng LC, Lu C. The clinical effect comparison between apatinib and S-1 as the second-line treatment for advanced gastric cancer. Oncol Prog. 2017;15(12):1436-1438.

31. Wang DP, Song SS, Wang K. Clinical effect analysis of apatinib mesylate combined with chemotherapy in the treatment of patients with advanced gastric cancer. World Latest Med Inform. 2016;16(87):130-131.

32. Wen F, Xiang Y, Wang L. Clinical trial of apatinib tablets in the treatment of advanced gastric cancer. Chin J Clin Pharmacol. 2017;33(7): 589-591.

33. Wu ZW, Cai J, Lu WK, Ma D, Hou J. Clinical study of Apatinib combined with Tegafur as second line treating advanced gastric cancer. China Mod Med. 2017;24(23):69-71.

34. Yan XH, Zhao YN, Wang H, et al. Efficacy observation of apapatinib combined with oxaliplatin and S-1 for treatment of advanced gastric cancer. Cancer Res Clin. 2017;29(11):761-764.

35. Zhan ZX. Short-term efficacy and prognosis analysis of apatinib combined with irinotecan and 5-FU on patients with advanced gastric cancer. Chin J Pract Med. 2017;44(13):39-42.

36. Zhou L, Sun YS, Wang XL, et al. Clinical observation of Apatinib combined with Tegafur in treatment of advanced gastric cancer patients after failure of second-line or beyond treatment. Contemp Med. 2018;24(5):52-54

37. Zhu HY, Sun XF, Zhou Q, Chen LX, Chen J, Wu PP. Clinical study of Apatinib combined chemotherapy in the treatment of advanced gastric cancer. Chin J Surg Oncol. 2016;8(6):394-396.

38. Zeng X, Zhang Y, Kwong JS, et al. The methodological quality assessment tools for preclinical and clinical studies, systematic review and meta-analysis, and clinical practice guideline: a systematic review. J Evid Based Med. 2015;8(1):2-10.
39. Jackson D, White IR, Riley RD. Quantifying the impact of between-study heterogeneity in multivariate meta-analyses. Stat Med. 2012;31(29): 3805-3820.

40. Han B, Jin B, Chu T, et al. Combination of chemotherapy and gefitinib as first-line treatment for patients with advanced lung adenocarcinoma and sensitive EGFR mutations: a randomized controlled trial. Int $J$ Cancer. 2017;141(6):1249-1256.

41. Fountzilas C, Chhatrala R, Khushalani N, et al. A phase II trial of erlotinib monotherapy in advanced pancreatic cancer as a first- or secondline agent. Cancer Chemother Pharmacol. 2017;80(3):497-505.

42. Li J, Qin S, Xu J, et al. Randomized, double-blind, placebo-controlled phase III trial of apatinib in patients with chemotherapy refractory advanced or metastatic adenocarcinoma of the stomach or gastroesophageal junction. J Clin Oncol. 2016;34(13):1448-1454.

43. Li J, Qin S, Xu J, et al. Apatinib for chemotherapy-refractory advanced metastatic gastric cancer: results from a randomized, placebo-controlled, parallel-arm, phase II trial. J Clin Oncol. 2013;31(26):3219-3225.

44. Zou K, Yang S, Zheng L, Yang C, Xiong B. Efficacy and safety of target combined chemotherapy in advanced gastric cancer: a meta-analysis and system review. BMC Cancer. 2016;16(1):737.

45. Chen J, Zhou SJ, Zhang Y, et al. Clinicopathological and prognostic significance of galectin-1 and vascular endothelial growth factor expression in gastric cancer. World J Gastroenterol. 2013;19(13):2073-2079.

46. Kerbel RS. Tumor angiogenesis. N Engl J Med. 2008;358(19): 2039-2049.

47. Poh SL, Linn YC. Immune checkpoint inhibitors enhance cytotoxicity of cytokine-induced killer cells against human myeloid leukaemic blasts. Cancer Immunol Immunother. 2016;65(5):525-536.

48. Dai C, Lin F, Geng R, et al. Implication of combined PD-L1/PD-1 blockade with cytokine-induced killer cells as a synergistic immunotherapy for gastrointestinal cancer. Oncotarget. 2016;7(9):10332-10344.

49. Zhang T, Cao L, Xie J, et al. Efficiency of CD19 chimeric antigen receptormodified T cells for treatment of B cell malignancies in phase I clinical trials: a meta-analysis. Oncotarget. 2015;6(32):33961-33971.

50. Katz SC, Burga RA, McCormack E, et al. Phase I hepatic immunotherapy for metastases study of intra-arterial chimeric antigen receptormodified T-cell therapy for CEA+ liver metastases. Clin Cancer Res. 2015;21(14):3149-3159.
Drug Design, Development and Therapy

\section{Publish your work in this journal}

Drug Design, Development and Therapy is an international, peerreviewed open-access journal that spans the spectrum of drug design and development through to clinical applications. Clinical outcomes, patient safety, and programs for the development and effective, safe, and sustained use of medicines are the features of the journal, which

\section{Dovepress}

has also been accepted for indexing on PubMed Central. The manuscript management system is completely online and includes a very quick and fair peer-review system, which is all easy to use. Visit http://www.dovepress.com/testimonials.php to read real quotes from published authors. 